\section{Von der Diakonie zum Sozialunternehmen}

Machen Sozialstaat und staatliche Leistungsaufträge die Diakonie überflüssig? Ein Deutungsversuch am Beispiel der Stiftung Schürmatt

\section{Christoph Weber-Berg}

Die Stiftung Schürmatt ist im Jahr 2018 eines der bedeutendsten Sozialunternehmen im Kanton Aargau. ${ }^{1}$ Über 500 Menschen mit Beeinträchtigungen wohnen, gehen zur Schule oder arbeiten in Einrichtungen der Schürmatt. Sie werden von mehr als 400 Mitarbeiterinnen und Mitarbeitern begleitet und unterstützt. Die Schürmatt ist heute eine konfessionell und politisch neutrale Stiftung und einzig die Tatsache, dass der Stiftungsrat durch die Synode und den Kirchenrat der Reformierten Landeskirche Aargau gewählt wird erinnert noch daran, dass die Schürmatt im Jahr 1963 als diakonisches Werk der Kirche gegründet wurde. Eine stattliche Anzahl sozialer Werke, die heute als unabhängige Stiftungen verfasst sind und über Leistungsverträge weitgehend vom Staat finanziert werden, weisen eine ähnliche Geschichte auf.

Es gibt auch Werke wie die Stiftung Satis in Seon- ${ }^{2}$ Sie wurde nicht von der Kirche gegründet, sondern ging im Jahr 1956 aus der privaten Initiative eines Ehepaars hervor, das Männern «in alkoholbedingten Notlagen» eine Aufnahme bieten sollte. Heute arbeitet die Stiftung mit Menschen mit psychischen Beeinträchtigungen, Personen im Massnahmenvollzug oder solchen im so genannten «Arbeitsexternat», der letzten Stu-

1 URL: www.schuermatt.ch, abgerufen am 10. April 2018.

2 URL: www.satis-seon.ch, abgerufen am 10. April 2018.

Jahrbuch Diakonie Schweiz 2 (2018) - ISSN 2504-3994

Dieser Text ist lizenziert unter einer Creative Commons Namensnennung 4.0 International Lizenz (CC BY 4.0): (https://creativecommons.org/licenses/by/4.0/). fe vor der bedingten Entlassung aus dem Strafvollzug. Von Anfang an bestand zwar ein enger Kontakt zur Reformierten Landeskirche, welche das Patronat über die Initiative übernahm. Doch erst 1969 wurde eine Stiftung errichtet, die der Landeskirche insofern anvertraut wurde, dass auch hier der Kirchenrat den Stiftungsrat einsetzt. Eine weitergehende Einflussnahme auf die Tätigkeit der Stiftung findet vonseiten der Kirche jedoch nicht statt. Es würde zu weit führen, eine Liste aller diakonischen, bzw. sozialen Werke zu erstellen, die entweder durch die Kirche gegründet oder ihr in irgendeiner Weise «anvertraut» wurden.

Verbindend ist diesen Werken, dass sie im Geist christlicher Liebestätigkeit gegründet und während vielen Jahren betrieben wurden, heute aber höchstens noch lose Verbindungen zur Kirche haben. Immer wieder hat sich die Kirche als Geburtshelferin oder Patin diakonischer Werke hervorgetan, die in einer späteren Phase ihrer Existenz auf eigenen Füssen standen und losgelöst, selbständig im Rahmen von Leistungsvereinbarungen mit dem Staat, ihre Aufgaben wahrnahmen. Im Kanton Aargau gibt es aktuell bloss wenige Ausnahmen von Einrichtungen, die nach wie vor eine institutionelle Bindung zur Kirche haben:

Einerseits die Heimgärten Aarau und Brugg, welche Frauen mit besonderen Betreuungsbedürfnissen (Schwerpunkt psychische Beeinträchtigungen) Aufnahme bieten. ${ }^{3}$ Die beiden Heimgärten sind nach wie vor Einrichtungen der Reformierten Landeskirche Aargau und nicht als Stiftungen verselbständigt. Als «unselbständige Anstalten öffentlichen Rechts» sind jedoch auch sie gegenüber dem Staat voll handlungsfähig und erbringen ihre Leistungen wie selbständige Stiftungen auf der Basis von Leistungsverträgen. In der täglichen Arbeit haben sie die sicht- und erlebbare Verbindung zur Kirche weitgehend abgestreift und unterscheiden sich in dieser Hinsicht nicht von verselbständigten Stiftungen.

3 URL: www.heimgarten-aarau.ch / www.heimgarten-brugg.ch, abgerufen am 10. April 2018. 
Eine weitere Ausnahme ist die von der Reformierten Landeskirche aufgebaute, und inzwischen von den drei Landeskirchen ${ }^{4}$ des Kantons getragene Palliative Care-Arbeit. In ihrem Rahmen werden Berufsleute und Freiwillige für die Begleitung schwer kranker oder sterbender Menschen und ihrer Angehörigen ausgebildet und es wird eine kantonale Einsatzzentrale betrieben, welche die Einsätze der Mitglieder freiwilliger Begleitgruppen koordiniert und Notfalleinsätze organisiert. Aus kirchlicher Perspektive ist diese Arbeit im Dreieck zwischen Pflege, Seelsorge und sozialer Arbeit im Wesentlichen als diakonische Arbeit zu verstehen. Die Palliative Care-Arbeit im Aargau ist darüber hinaus ein Beispiel dafür, dass es auch heute möglich ist, dass kirchliche Diakonie gesellschaftliche Pionierarbeit leistet. Während der Kanton den Vorgaben des Bundes in diesem Bereich weiterhin hinterher hinkt, hat die Kirche das Thema schon vor vielen Jahren aufgegriffen und sich als Partnerin von Kliniken, Spitälern und weiteren Institutionen einen Namen geschaffen.

Die dritte Ausnahme ist die Inlandarbeit des Hilfswerks der Evangelischen Kirchen der Schweiz HEKS, welche von der Landeskirche und von Kirchgemeinden weiterhin finanziell unterstützt wird. Im Kanton Aargau ist es so, dass die diakonische Arbeit der Landeskirchlichen Dienste faktisch an die Regionalstelle Aargau-Solothurn des HEKS ausgelagert wird. Die Landeskirchliche Fachstelle Diakonie arbeitet vorwiegend in Bereichen wie Aus- und Weiterbildung oder Koordination diakonischer Projekte in Gemeinden. Auch wenn HEKS für diejenigen, die genau hinschauen, als kirchliches Hilfswerk erkennbar ist, so spielt die Kirchlichkeit der Institution in ihrer täglichen Arbeit mit Klientinnen und Klienten keine unmittelbar sichtbare Rolle mehr. Auch HEKS generiert einen beachtlichen Teil seiner Erträge durch Leistungsvereinbarungen mit dem Staat.

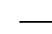

4 Neben der Reformierten Landeskirche sind das die Römisch-katholische Kirche im Aargau sowie die Christkatholische Landeskirche Aargau.
Die Ausnahmen bestätigen im Grunde die Regel, welche dahingehend $\mathrm{zu}$ formulieren ist, dass institutionalisierte kirchliche Diakonie in der zweiten Hälfte des 20. Jahrhunderts einen kräftigen Aufschwung erfahren, und sich gegen Ende des Jahrhunderts von der Kirche weitgehend emanzipiert hat.

Ich möchte diese Entwicklung in der Folge kurz am Beispiel der Stiftung Schürmatt nachzeichnen, um danach eine theologische Einordnung zu versuchen.

Die Initiative zur späteren Gründung der Stiftung Schürmatt geht in die Fünfzigerjahre zurück ${ }^{5}$. Damals bestanden nur wenige Möglichkeiten, Kinder mit geistigen Behinderungen in professionell geführten Einrichtungen wohnen und zur Schule gehen zu lassen. Die wohl grösste Einrichtung, welche nach damaligen Kriterien auch «nicht schulbildungsfähige» Kinder aufnahm, war das katholische St. Josefsheim in Bremgarten. Für den reformierten Fricktaler Bauern und Kirchenpfleger Fritz Hodler und seine Frau Rosa, die zwei Töchter mit Behinderungen hatten, kam es damals nicht in Frage, ihre Kinder in ein katholisches Heim zu geben. Sie brachten ihren Gemeindepfarrer Hans Schneider dazu, in der Sommersynode 1956 eine Motion einzureichen, welche den Kirchenrat verpflichtete zu prüfen, ob die Landeskirche etwas dagegen tun könne, dass reformierte Kinder in ein katholisches Heim gegeben werden müssten. Die Landeskirche sollte prüfen, ob sie aus ihren Mitteln, eventuell zusammen mit Partnern, «diesen Mangel beheben» könne. Eine vom Kirchenrat eingesetzte Kommission sollte in der Folge die Möglichkeit prüfen, ob

5 Wo nicht anders vermerkt, sind die Informationen über die Entstehungsgeschichte der Schürmatt dem Band «Wie die Schürmatt entstand» (1996), von Kurt Walti entnommen. Walti war Kirchenratssekretär der Reformierten Landeskirche Aargau in den Jahren 1974 bis 1994. Er wirkte während rund 30 Jahren in Planung, Aufbau und Leitung der Schürmatt mit. 
das Reformierte Kinderheim Brugg entsprechend erweitert werden könnte. Die Kommission scheint ihre Arbeit verschleppt zu haben; jedenfalls dauerte es bis 1960, bis Bewegung in das Anliegen der Motion Schneider kam. Damals wurde auf nationaler Ebene beschlossen, dass sich die Schweizerische Invalidenversicherung IV mit Subventionen an der Errichtung von Heimen für Menschen mit geistigen Behinderungen beteiligen werde. Im Frühjahr 1960 wurde Pfarrer Kurt Walti mit der Ausarbeitung eines Vorprojektes beauftragt und an der Sommersynode liess sich der Kirchenrat die Kompetenz erteilen, einen Landkauf vorzubereiten und der Synode zur Genehmigung vorzulegen. Schon im August 1960 war der Kauf des Grundstücks Schürmatt in Zetzwil vom Kirchenrat beschlossen. Sehr rasch machte sich in der Folge eine kirchenrätliche Planungskommission an die Arbeit, die das zukünftige Kinderheim unter Beizug von Expertinnen und Experten konzipierte und plante.

Die Stiftungsgründung erfolgte im Jahre 1963, der Bau wurde in den Jahren 1964-65 realisiert, und Anfang Juli 1965 zogen die ersten Kinder ein.

Kurt Walti identifizierte in seinem 1996 verfassten Rückblick auf die Gründerjahre der Schürmatt eine Reihe von Erfolgsfaktoren. Als wesentlichen Punkt hebt er das gute Zusammenspiel zwischen Kirchenrat und Synode hervor. Nachdem die Synode die Motion «einmütig» überwiesen hatte, kam offensichtlich auch im weiteren Verlauf des Projekts wenig substanzielle Opposition aus dem Kirchenparlament. Ein Argument von Opponenten war theologischer Art und verwies darauf, dass die Verkündigung primäre Aufgabe der Kirche sei, und nicht etwa der Aufbau und die Führung eines Kinderheims. Das Argument verfing jedoch nicht angesichts des ebenfalls theologischen Gegenarguments, dass diakonisches Engagement ebenfalls verkündigenden Charakter habe und darüber hinaus die Wortverkündigung in ihrer Glaubwürdigkeit bestärke. Die Synode vom 15. Mai 1963 beschloss jedenfalls im Stimmenverhältnis 10:1 nicht nur die Gründung der Stiftung Schürmatt, sondern auch, ei- nen Gründungsbeitrag von maximal 1.6 Mio $\mathrm{CHF}^{6}$ sowie jährliche Betriebsbeiträge im Rahmen des landeskirchlichen Budgets zu leisten.

Als weitere Erfolgsfaktoren identifizierte Kurt Walti das gute Verhältnis zu Kirchgemeinde und politischer Gemeinde am Standort Zetzwil sowie die persönlichen Beziehungen des damaligen Kirchenratssekretärs Armin Byland zur Kantonsregierung (Regierungsrat Kurt Kim war zur fraglichen Zeit sogar Mitglied des Kirchenrates) und zu Chefbeamten der kantonalen Verwaltung.

Als wichtiger Erfolgsfaktor nicht unerwähnt bleiben darf das $\mathrm{Zu}$ sammenwirken des charismatischen ersten Leiters der Schürmatt, Pfr. Hermann Wintsch, der - nach Waltis Aussage - «das schöpferische Chaos bevorzugte», mit seinen Kontaktpersonen der Landeskirche. Wintsch wurde von Kurt Walti und Hans Brack, dem Verwalter der Landeskirche während vielen Jahren eng begleitet. Diese drei Männer: der charismatische Heimleiter Wintsch, der strukturierende und ordnende Kirchenratssekretär Walti («für mich ist eine klare Organisation wie Luft zum Atmen») und der begnadete Fundraiser Brack (in den Worten Waltis: «bekannt für sein einnehmendes Wesen») scheinen die Pionierphase der Schürmatt wesentlich geprägt zu haben.

Ein wichtiger Meilenstein in der Geschichte der Schürmatt ist die Eröffnung des Bereichs «Erwachsene» im Jahre 1982. Aus dem Kinderheim wurde nach und nach ein Sozialunternehmen, das Menschen mit Behinderungen in allen Phasen des Lebens eine Heimat und einen Arbeitsoder Beschäftigungsort bieten konnte. Es entstanden Aussenstandorte mit Wohngruppen und Werkstätten, wobei den Aspekten der Integration und der Kooperation zwischen Menschen mit und ohne Behinderungen besondere Aufmerksamkeit geschenkt wurde. In diesem Zusammenhang

6 Nach heutiger Kaufkraft entspricht dieser Betrag knapp 6 Mio CHF. Vgl. LIKTeuerungsrechner von Statistik Schweiz, URL: http://www.portal-stat.admin.ch/lik _rechner/d/lik_rechner.htm, abgerufen am 14. März 2018. 
wurden zum Beispiel Werkstätten direkt in einem Industriebetrieb eingerichtet, womit Menschen mit und ohne Behinderung im täglichen $\mathrm{Ar}-$ beitsprozess zusammenarbeiten. Der kooperative Ansatz, der nicht nur dafür sorgt, dass Menschen mit Behinderungen ihren Bedürfnissen entsprechend begleitet und betreut werden, sondern auch, dass sie - zur gegenseitigen Bereicherung - mit Menschen ohne Behinderung niederschwellig und alltäglich zusammenarbeiten können, wird auch im Kindergartenbereich umgesetzt. Hier werden Kinder mit und ohne Behinderungen in parallelen Klassen unterrichtet, wobei einzelne Unterrichtsteile gemeinsam und einzelne getrennt abgehalten werden.

Die Stiftung Schürmatt verfügt heute im Kanton Aargau über einen ausgezeichneten Ruf als Sozialunternehmen. Das hat die Stadt Aarau im Jahre 2013 dazu veranlasst, der Schürmatt die Trägerschaft und den Betrieb ihrer Heilpädagogischen Sonderschule HPS anzuvertrauen. Dieser Schritt, zusammen mit der Übernahme des Auftrags zum Aufbau des Heilpädagogischen Zentrums für Werkstufe und Berufsvorbereitung, hat auch in der Öffentlichkeit einige Wellen geworfen. Im Wesentlichen zwei Gründe waren dafür verantwortlich: Einerseits die Frage, ob es verantwortbar sei, dass die Öffentliche Hand die HPS an eine private Trägerschaft abgebe. Die Bereitstellung und der Betrieb des heilpädagogischen Schulangebots sei genauso öffentliche Aufgabe wie das Angebot der Regelschule. Der Betrieb heilpädagogischer Schulen durch Private wirke der Integration von Kindern und Jugendlichen mit Behinderung in die Gesellschaft entgegen, wurde vor allem vonseiten der Lehrerschaft argumentiert. ${ }^{7}$ Das zweite Argument wurde ebenfalls vom Lehrerinnenund Lehrerverband ins Feld geführt: Man erinnerte sich daran, dass die Schürmatt fünfzig Jahre früher eine kirchliche Gründung gewesen war und befürchtete, dass die weltanschauliche Neutralität in der durch die

7 URL: https://www.srf.ch/news/regional/aargau-solothurn/aargauer-heilpaedago gische-schulen-zurueck-zur-volksschule, abgerufen am 29. März 2018.
Schürmatt oder andere private Trägerschaften betriebene HPS in Gefahr stehen könnte.

Die kantonalen Behörden teilten die Befürchtungen nicht und im Betrieb der ersten Jahre zeigte sich, dass sie auch gänzlich unbegründet waren. Eine externe Schulevaluation im Jahre 2017 attestierte der von der Schürmatt geführten HPS Aarau vorbehaltslos eine ausgezeichnete Qualität.

Die Entwicklung der Schürmatt über mehr als 50 Jahre ist nicht untypisch für kirchliche oder kirchennahe diakonische Werke. Sie spannt den Bogen von der christlich motivierten diakonischen Pioniertat bis zum modernen, ausdifferenzierten und professionell geführten Sozialunternehmen mit staatlichem Leistungsauftrag.

Diese Entwicklung geschah im Lauf einer Zeitspanne, welche durch die Jahre des Aufschwungs, der wirtschaftlichen Entwicklung und des wachsenden Wohlstands geprägt war. Spätestens ab den 60er Jahren waren die wirtschaftlichen Folgen der Kriegsjahre so weit überwunden, dass breite Bevölkerungsschichten von zunehmenden Konsumoptionen sowie wachsender individueller Mobilität und Freiheit profitieren konnten. Altersversicherung, Invalidenversicherung, Kranken- und Unfallversicherung, berufliche Vorsorge, Arbeitslosenversicherung und Sozialhilfe sorgten dafür, dass nebst steigenden Reallöhnen die gesamte Bevölkerung durch zunehmende soziale Absicherung am gesellschaftlich erarbeiteten Wohlstand teilhaben konnte. Dieser Umstand begünstigte die Institutionalisierung, Professionalisierung und Ausdifferenzierung der Begleitung und Betreuung von Menschen mit besonderen Bedürfnissen.

Diakonische Werke waren bis in die Mitte des zwanzigsten Jahrhunderts oft durch evangelisch-reformierte Diakonissen oder katholische Ordensleute geführt worden. Die oben genannte St. Josefsstiftung Bremgarten, welche sich um Kinder mit Behinderungen kümmerte, wurde von Ingenbohl-Schwestern geführt. Im Reformierten Kinderheim Brugg waren es während vielen Jahren Riehener Diakonissen, welche die anvertrauten Kinder betreuten. 
In die 60er Jahre aber, die Pionierphase der Schürmatt, fiel ein erster Professionalisierungsschub. Nicht, dass Ordensschwestern und Diakonissen grundsätzlich unprofessionell gearbeitet hätten, aber die christliche Hingabe des ganzen Lebens trat gegenüber den Ansprüchen an die Professionalität der mitarbeitenden Fachpersonen in den Hintergrund. Das wurde möglich, und ist für die Gründung der Schürmatt belegt, ${ }^{8}$ aufgrund der Tatsache, dass die Invalidenversicherung IV nicht nur Renten an Betroffene auszahlte, sondern auch den Bau und den Betrieb von Heimen subventionierte.

Weiterhin aber sahen es Kirchen als ihre Aufgabe an, sich besonders um die Betreuung von Menschen mit Behinderungen zu kümmern. Bezeichnenderweise wurden in der Pionierphase der Schürmatt Pfarrer mit Konzeption und Leitung des Kinderheims betraut. Im Zuge dieser Erstprofessionalisierung scheint es noch - vermutlich unausgesprochen - ein Anliegen gewesen zu sein, die Leitungskompetenz bei im weiteren Sinne kirchlich beauftragten Personen zu haben. Das änderte sich in der weiteren Aufbau- und Expansionsphase. Professionalisierung und Differenzierung der Aufgaben erreichten auch die Leitungsebene. Während Pfarrer Wintsch, «Heimleiten» der Pionierphase, noch mit seiner ganzen Familie in der Schürmatt lebte und nicht nur in der Heimleitung, sondern auch direkt an der Betreuung der Kinder beteiligt gewesen war, so waren und sind seine Nachfolger «Direktoren», ausschliesslich mit Leitungsfunktionen betraut, extern wohnhaft und ohne berufliche Sozialisation in der Kirche.

Auch im finanziellen Bereich fand diese Verschiebung statt. Vor der Gründungszeit der Schürmatt waren - idealtypisch und verkürzt gesagt die in der Betreuungsarbeit engagierten Personen Diakonissen oder

8 Kurt Walti in seinem Bericht von 1996 (Anm. 5): « ... und anfangs April 1960 berichteten die Medien, dass die IV auch Subventionen an die Errichtung von Heimen für Geistigbehinderte ausrichten werde» (3).
Nonnen, welche für Gotteslohn ihr ganzes Leben allen im Umfeld der Betreuung von Kindern mit Behinderung anfallenden Arbeiten widmeten. Die Kosten waren tief, die christliche motivierte Hingabe gross. Die damalige Gesellschaft konnte sich wohl oder übel andere Betreuungsformen kaum leisten. Ab den 60er Jahren aber wurden dank wirtschaftlichem Aufschwung und steigendem Wohlstand staatlich regulierte oder staatlich finanzierte Sozialwerke möglich, welche ihre Gelder unter der Auflage von Professionalitäts- und Qualitätsstandards an kirchliche Organisationen auszahlten. Kirchliche Investitionen und Defizitgarantien, wie sie durch die Reformierte Landeskirche Aargau anfänglich auch für die Schürmatt gesprochen wurden, wurden zunehmend überflüssig. Während in der Pionierphase Tisch- und Abendgebete in diesen Institutionen wohl kein Problem, ja sogar eher die Norm darstellten, so wurden im Lauf der Jahre zunehmend auch Anforderungen an weltanschauliche Neutralität an die Leistungsaufträge geknüpft.

Doch wie war es zur beschriebenen Gründer- und Pionierdynamik aus kirchlichem und kirchennahem Umfeld gekommen? Die 60er und 70er Jahre des letzten Jahrhunderts waren vor dem Hintergrund des wachsenden Wohlstands auch Jahre des Aufschwungs für die Kirche. Einerseits waren damals Kirchenaustritte noch praktisch kein Thema. Andererseits waren die kirchlichen Finanzetats tendenziell steigend. Es wurden Neubauten erstellt und auch in der Kirche fand ein Professionalisierungsschub statt. Die Anzahl Angestellter in Kirchgemeinden, kirchlichen Verwaltungen und Landeskirchlichen Diensten nahm deutlich zu. Das gesellschaftliche Selbstbewusstsein der Kirche war nach wie vor hoch und man mutete sich zu, etwas bewegen zu können. Anders ist die Gründung von Werken wie der Schürmatt nicht zu denken. Das finanzielle Engagement der Landeskirche und der Kirchgemeinden im Rahmen der Gründung und des Aufbaus der Schürmatt war signifikant und wäre proportional in gleicher Höhe wohl heute kirchenpolitisch nur noch schwer mehrheitsfähig. Es fehlt heute der damals verbreitete Geist des Aufschwungs und des Fortschrittsoptimismus. 
Die Kirche hat sich heute weitgehend aus dem Betrieb und der Finanzierung diakonischer Werke zurückgezogen. Der Betrieb wurde professionalisiert, die Finanzierung verstaatlicht. Um die oben verwendete, zuspitzende Diktion aufzunehmen: Die christliche Hingabe ist tief, die Kosten sind hoch.

Eine theologische oder kirchenpolitische Beurteilung dieser Entwicklung darf nicht $\mathrm{zu}$ allzu schnellen Schlüssen zwischen «gut» oder «schlecht» kommen, denn das primäre Ziel der qualitativ hochstehenden Begleitung und Betreuung von Menschen mit besonderen Bedürfnissen wird heute auf einem ganz anderen Niveau sichergestellt als vor fünfzig Jahren.

Sachlich kann festgestellt werden, dass sich mit dem wirtschaftlichen Aufschwung nach dem Krieg zunächst auch für die Kirche neue Möglichkeiten eröffneten, diakonische Arbeit zu professionalisieren und zu institutionalisieren. Gleichzeitig wurden staatliche Sozialwerke wie die Invalidenversicherung auf- und ausgebaut, was die Möglichkeit bot, kirchlich-diakonischen Anliegen Schub zu verleihen. Die Schürmatt, deren Gründung direkt mit der 1960 beschlossenen Subventionierung des Aufbaus von Heimen für Menschen mit Behinderung durch die IV in Verbindung gebracht werden kann, gibt davon unmittelbares Zeugnis. Der Auf- und Ausbau des Sozialstaates sowie die allgemeine Säkularisierung der Gesellschaft führten jedoch bald dazu, dass die Sicherstellung von Angeboten für Menschen mit Beeinträchtigungen zunehmend als staatliche Aufgabe betrachtet wurde. Emanzipation von Menschen mit Behinderung aus enger Bevormundung sowie deren Integration und Partizipation am gesellschaftlichen Leben wurden zu sozialen Anliegen, die keiner religiösen Motivation oder Begründung mehr bedurften. Gleichzeitig wuchs mit der Säkularisierung eine in den meisten Fällen zwar unbegründete, aber nicht wegzudiskutierende Skepsis gegenüber kirchlich getragenen Werken. Es wurde wichtig gegenüber der Öffentlichkeit zu betonen, dass sie weltanschaulich neutral geführt, keinerlei religiöse Beeinflussung von Klientinnen und Klienten betreiben. Dass diese Skepsis bis heute nicht ganz verschwunden ist, zeigt die oben be- schriebene, ablehnende Haltung aus Kreisen des Lehrpersonals gegen die Übernahme der Heilpädagogischen Sonderschule Aarau durch die Schürmatt.

Aus kirchenpolitischer Sicht ist diakonisches Engagement im Namen und Auftrag der Kirche nach wie vor durchaus zu begrüssen. Wie schon in der Synode 1960 argumentiert wurde, stärkt es die Glaubwürdigkeit kirchlicher Verkündigung. Es stärkt in gewisser Weise auch die Position der Kirche in einer säkularisierten Gesellschaft, indem es als Argument für den allgemein-gesellschaftlichen Nutzen der Existenz und des Wirkens der Kirche herbeigezogen werden kann. Nicht zuletzt aus diesem Grund ist in kirchlichen Kreisen der Wunsch immer wieder zu hören, doch nicht alle diakonischen Werke in unabhängige Stiftungen zu überführen und es wird gleichzeitig bedauert, dass kirchlich getragene oder mitfinanzierte Sozialwerke in der öffentlichen Wahrnehmung kaum noch mit der Kirche in Verbindung gebracht werden.

Allerdings erfassen kirchenpolitische oder reputationsstrategische Überlegungen nicht den Kern dessen, worum es aus theologischer Sicht geht. Dieser lässt sich mit zwei Fragen umschreiben, die in einem engen inneren Zusammenhang stehen:

- Was ist der Unterschied zwischen sozialer Arbeit oder sozialem Engagement und Diakonie?

- Welche Bedeutung hat die Diakonie für das Kirche-Sein?

Aus einem sozialen Projekt wird nicht automatisch ein diakonisches Projekt, wenn es von der Kirche vollständig oder teilweise finanziert wird. Nicht die Geldflüsse oder der Ursprung von Geldflüssen machen den theologisch formulierbaren Unterschied aus. Es ist auch nicht der Umstand, dass ein soziales Werk einmal von der Kirche oder von christlich motivierten Menschen gegründet wurde. Der Unterschied manifestiert sich an der Frage, welche Bedeutung ein soziales Werk für Menschen hat, die miteinander Kirche sein wollen. Die ehemalige Genfer Synodalratspräsidentin Charlotte Kuffer formulierte es mündlich einmal treffend: «Diakonie ist, wenn dahinter (hinter einem sozialen Werk) eine betende Gemeinde steht». Es ist demnach nicht nur die christliche Moti- 
vation einzelner sozial tätiger Menschen, es sind das Gebet und die Gemeinschaft der Betenden, die aus sozialer Arbeit diakonisches Engagement machen. Die Bedeutung der Diakonie für das Kirche-Sein geht weit über Reputationsfragen und Kirchenpolitik hinaus: Sie wurzelt in der Fürbitte der Gemeinde. Fürbitte ohne diakonisches Engagement läuft ins Leere. Und diakonische Arbeit ohne Fürbitte unterscheidet sich kaum von anderer - ebenfalls und unzweifelhaft wertvoller - sozialer Arbeit.

Für eine Landeskirche wie die Aargauische stellt sich in diesem Zusammenhang die Frage, was dies angesichts schwindender finanzieller Ressourcen bedeutet. Ist den Verantwortlichen in Gemeinden, in der Synode und im Kirchenrat bewusst, welche Bedeutung die Diakonie für die Beurteilung des eigenen Kirche-Seins hat? Mit der Finanzierung sozialer Anliegen und Werke allein ist es nicht getan. Zugespitzt könnte man dies sogar als «institutionellen Ablass» verstehen. Umgekehrt stellt der Rückzug aus der Finanzierung sozialdiakonischer Projekte und Werke potenziell das eigene Kirche-Sein in Frage.

Diakonie ist kirchliche Verkündigung durch die Tat. Modern gesprochen «performative Kommunikation». Die Performance stärkt die Glaubwürdigkeit der Kommunikation, bzw. Diakonie die Glaubwürdigkeit der Predigt. Das Mittragen sozialer Anliegen im Gebet als Gemeinde ist zentral für deren Kirche-Sein.

Es kommt dabei nicht auf die Höhe der verteilten Geldsummen oder der diakonisch eingesetzten Zeit von Personen an. Es heisst also nicht, dass Sparen bei diakonischen Anliegen grundsätzlich verboten sein sollte. Diakonie ist nicht fälschlicherweise als Versuch zu verstehen, sich als Kirche oder als Mensch vor Gott durch die Liebestat demonstrativ zu rechtfertigen. Dass wir aus lauter Gnade gerettet sind, gilt auch in diesem Zusammenhang. Entscheidend ist, ob eine Gemeinde oder eine Landeskirche das, was sie durch die eigene Tat oder durch Geldspenden unterstützt, auch im Gebet mitträgt und als Teil des ganz eigenen KircheSeins verstehen lernt.
Dann ist plötzlich weniger wichtig, ob ein ehemals kirchliches Werk heute unabhängig und konfessionell neutral unterwegs ist oder nicht. Entscheidend ist dann, dass das durch dieses Werk verwirklichte Anliegen aus dem Glauben einzelner Menschen und im Gebet der Gemeinde mitgetragen wird.

Diakonie wird also nicht überflüssig, wenn der Staat ehemals diakonische Anliegen und Werke über Leistungsverträge mit unabhängigen Stiftungen finanziert. Diakonie ist keine Frage der Institutionalisierung und Finanzierung sozialer Werke und Anliegen. Sie ist eine Frage der Haltung, aus der heraus Menschen gemeinsam Kirche sein wollen.

Wie das Beispiel Palliative Care zeigt, kann ausserdem diakonische Arbeit der Kirche immer wieder Pionierarbeit in Bereichen sein, die später als staatlich zu finanzierende Aufgaben erkannt werden.

Autor:

Christoph Weber-Berg,

Pfr. Dr. theol., Kirchenratspräsident der Reformierten Landeskirche Aargan 